\title{
Writer Identification by Professional Document Examiners ${ }^{\dagger}$
}

\author{
Moshe Kam, ${ }^{*}$ Ph.D.; Gabriel Fielding, ${ }^{*}$ M.Sc.; and Robert Conn, ${ }^{* *}$ Ph.D. \\ * Data Fusion Laboratory, \\ Electrical and Computer Engineering Department, \\ Drexel University, \\ Philadelphia, PA 19104 \\ and \\ ** Applied Physics Laboratory \\ The Johns Hopkins University, \\ Baltimore, MD 20723
}

\begin{abstract}
Reliable data on the capabilities of professional document examiners are scarce, rendering most past characterizations of these capabilities somewhat speculative. We report on a comprehensive test administered to more than 100 professional document examiners, intended to close this data gap in the area of writer identification. Each examiner made 144 pair-wise comparisons of freely-created original handwritten documents. The task was to determine whether or not a "match" was detected, namely whether or not the two documents were written by the same hand. Matching criteria were based on the identification and strong probability definitions of the ASTM standard E1658. The professionals were tested in three groups (in the northeastern, southeastern, and southwestern United States). In addition, we have created a control group of similar educational background. Several individuals training to become professional document examiners were tested as well.

Examination of the data and statistical tests show that the answers collected from the professional and nonprofessional groups came from different populations. The trainees' data were shown to have come from a population that is distinct from both professional and nonprofessional groups. Unlike the professional examiners, the nonprofessionals tended to grossly over-associate. They erroneously "matched" many documents that were created by different writers, mismatching almost six times as many unknown documents to database documents as the professionals did (38.3\% vs. $6.5 \%$ of the documents).

The results of our test lay to rest the debate over whether or not professional document examiners possess writer-identification skills absent in the general population. They do.
\end{abstract}

Keywords: forensic science, document examination, proficiency testing, writer identification, handwriting analysis, handwriting tests, questioned documents, document examiners

\footnotetext{
${ }^{\dagger}$ The study was funded by the Federal Bureau of Investigation and executed by the Data Fusion Laboratory at the Department of Electrical and Computer Engineering of Drexel University. We are grateful to the many individuals and professional groups that helped us in administering and conducting the test.
} 


\section{Introduction}

The area of document examination has experienced a heightened level of debate on examiner proficiency since the publication of a controversial paper by Risinger, Denebeaux and Saks in 1989 [1]. The discussion, in technical journals, professional meetings, and court hearings, has been characterized by acute lack of empirical evidence on the proficiency of document examiners. Indeed the only test performed so far with a control group was our own ([2], 1994). Lacking a meaningful body of data from controlled experiments, the proficiency debate has centered on refutation of uncontrolled tests ([1], [2, p. 6]), various attempts to aggregate and extrapolate old data (e.g., [1], [3]), refutations of past attempts to analyze old data [3] , and general discussions of document examination methodology and document-examiner certification (e.g., [4]). The proficiency of professional document examiners was also discussed in various court proceedings (e.g., [5] - [12]). While opinions on proficiency of human document examiners still vary, it is widely agreed that testing of professional document examiners and acquiring data on their abilities (compared to those of non-professionals) are necessary. Our previous study [2] was a small step in this direction. The present study is a very much larger step.

From May to September 1996, we conducted proficiency tests of more than one hundred American questioned-document examiners, in three groups of about 35 each: the Northeastern group (34 individuals tested in New York City and Washington DC); the Southeastern group (34 individuals tested in Atlanta, Georgia); and the Southwestern group (37 individuals tested in Reno, Nevada). In addition we have tested 41 non-experts 
in Philadelphia — we organized this group to resemble the professional groups in term of formal education (high-school/BA-BS/graduate degrees). A fifth group of eight subjects consisted of individuals who were in training to become professional document examiners. The total number of professional document examiners that participated in our test is estimated to be between one-sixth and one-third of the entire community of professional document examiners in the United States.

The test consisted of comparing pairs of documents and deciding whether or not they have been created by the same hand. Each test-taker was given two packages. The first package contained six original handwritten documents. We shall refer to it as the "unknown package" and to its documents as the "unknown documents". The second package contained twenty-four original handwritten documents - we shall call it the "database package" and call its documents the "database documents". The task was to find, for each one of the unknown documents, all the documents in the database package that are matches, in the sense that the test-taker can declare "identification" or "strong probability" of matching. The terms "identification" and "strong probability" were defined per ASTM standard E1658 [13].

The first goal of our study was to decide whether the test-score samples that we received from the groups of professionals and nonprofessionals come from the same population or from different populations. The second goal was to determine the absolute performance of the participating groups, under the constraints of the tests. 


\section{Summary of the Main Results}

- The results of our test establish firmly that the samples obtained from the professional groups and the samples obtained from the nonprofessional group came from distinctly different populations. In other words, the matching decisions made by the professionals were statistically different from the matching decisions made by the nonprofessionals. We have established this result with respect to five scoring criteria for the data, and two types of nonparametric statistical procedures: (i) procedures based on ranks of scores and (ii) procedures based on distributions of scores.

- The group of professionals incorrectly matched $\mathbf{6 . 5 \%}$ of the documents in the unknown packages with documents in the database packages. The group of nonprofessionals made this mistake for $\mathbf{3 8 . 3 \%}$ of the documents in the unknown packages.

- Nonprofessionals in our test tended to "over-associate" indiscriminately. As a result they found as many correct matches as the professionals did - but have declared many non-matching pairs to be matches.

- There were no significant statistical differences among the samples that came from the three professional groups.

- The sample generated by the trainees was significantly different from the sample generated by the professionals.

- The sample generated by the trainees was significantly different from the sample generated by the nonprofessionals. 


\section{The Test-takers}

The professional test-takers were either currently-employed or recently-retired professional questioned-document examiners, employed by law enforcement agencies or in for-profit private practice. The overwhelming majority of professional test-takers (99 out of 105) responded in full to voluntary questionnaires attached to the tests, identifying themselves by name, describing their education and training, and providing detailed accounts of their professional histories and professional duties. Almost all of the professional test-takers that answered our questionnaires were certified by, or were members of, one or more of the following organizations: American Academy of Forensic Sciences - Questioned Documents Section; American Board of Forensic Document Examiners; Southeastern Association of Forensic Document Examiners; Southwestern Association of Forensic Document Examiners; and the American Society of Questioned Document Examiners. Members of the Northeastern group were individually invited to the exam using lists of examiners residing in the vicinity of New York and Washington DC who are members of these professional organizations. Members of the Southwestern and Southeastern groups were attendees of the May 1996 professional meetings of questioned-document examiner regional associations.

The non-professional test-takers were students and educators from the Greater Philadelphia area, 34 of them holders of college degrees (B.A., B.Sc., M. A., M.Sc., M.Ed, MBA, Ph.D.) in Engineering, Education, or Management. The other seven were senior undergraduate students in Engineering. The non-professionals were screened for education level in order to match the educational profile of the professional groups. To induce their best efforts, non-professional test-takers were paid for their time, and were rewarded with financial performance-based incentives. Test-takers received $\$ 25$ for participation and $\$ 25$ for each correct match. We subtracted $\$ 25$ for each incorrect 
match, and $\$ 10$ for each missed match. If the resulting payment was less than $\$ 25$, the participant received $\$ 25$. The average test that we administered to nonprofessionals would have netted a payment of $\$ 102$ for a perfect score (plus $\$ 25$ for participation); some of our tests could yield a payment in excess of $\$ 200$.

\section{The Documents}

\section{Data Collection}

We have collected 1800 original handwritten documents generated by a group of 150 writers of ages 20-27, working on wide and well-lit tables in a classroom setting. Each writer generated 12 documents on $8^{1} / 2$ " x 11", $20 \mathrm{lb}$. white pages, copying three given texts four times each. We have supplied the paper and the writing utensils, blue and black medium-tip Bic pens. We instructed the writers to switch pens every 2-3 documents so that both 'blue' and 'black' documents be created.

The following texts were used:

1. The claim of their lawyers was simple. No one got hurt, the police framed the alleged stick up perpetrators, the dye packs stopped the defendants, and the alarms went off before any dollar bills changed hands.

2. No, these were not the people who would commit a robbery, use bombs or guns. But in their lust for money these administrators have done even worse. They have betrayed the public trust. 
3. His progress was slow, but at the end his persistence paid off. Many of his classmates were deemed brighter, more promising. But at the end of the day, he has surpassed them all, using the most potent weapons - dogged pursuit; eyes always on the prize; nobody, nothing ever capable of throwing him off the track or dampening his spirit.

\section{Data Organization}

We have created five 360-document sets - each was generated by 30 distinct writers. Each set was labeled by a letter $A$ through $E$.

The 360 documents of set $A$ were assigned random numbers; a key, associating the random numbers with their writers, was created and secured. Twelve packages of six documents were created and marked unknown (thus creating unknown A1, unknown A2, ..., unknown A12). The unknown documents in each package were selected randomly from set $A$, under the condition that six distinct writers be represented in each unknown package. The remaining 288 documents were randomly grouped into twelve database packages with 24 documents each (thus creating database A1, database A2, ..., database A12).

This procedure was repeated for sets $B, C, D$, and $E$. Every test-taker was provided with an unknown package and a database package, both from the same 360-document set (e.g., unknown D3 - database D12).

\section{Security}

In order to frustrate hypothetical (and highly-unlikely) individual attempts to use recorded answers from one test in a later test, we have changed the pairing of tests in every session. 
Thus if the pairing unknown Al - database Al was used in New York it was never used in Washington, Atlanta or Reno. Every time unknown Al was used again, it was paired with another database-set from the $A$ group. Even if the correct results of an early test were fully known to all test-takers in a later session, this information was practically useless. We do not believe that any attempt was made to record or share results from our tests between test-takers. However, even if such attempts were made, they could not affect the results in a meaningful way.

The processing and preparation of the document packages and the solution key were executed by a team headed by the second author. This team did not include the first author who administered the tests in New York, Washington DC, Reno, and Atlanta. The solution key (that associated the document identification number with its writer) was secured by the second author in Philadelphia. It was not shipped or communicated to any other individual or group, including the first author, until the tests were completed. The key was not available physically or electronically to anyone in New York, Washington DC, Reno, or Atlanta, including the first author who conducted the tests there.

\section{Statistics on the Documents}

Every test-taker received an unknown package of six documents and a database package of 24 documents. We have compiled a set of statistics about all possible pairings of tests. These statistics are: the percentage of tests for which $m$ unknown documents had matches, the percentage of unknown documents with $m$ matches, and the total number of matches per unknown package.

Table 1 shows the percentage $p$ of tests where $m$ unknown documents had matches (here $m=0,1,2,3,4,5,6)$. The third row of the table reads: "in $15.97 \%$ of the tests exactly 
two of the six documents in the unknown packages had a match or matches in the corresponding database packages."

\begin{tabular}{c|c}
\hline $\boldsymbol{m}$ unknown documents & $\boldsymbol{p}$, percentage \\
\hline 0 & $0.00 \%$ \\
1 & $3.47 \%$ \\
2 & $15.97 \%$ \\
3 & $36.11 \%$ \\
4 & $27.09 \%$ \\
5 & $15.97 \%$ \\
6 & $1.39 \%$ \\
\hline
\end{tabular}

Table 1: In $p$ percent of the tests, $m$ documents in the unknown package had matches in the database package

The table shows that each test-taker was expected to find matches for at least one document. The most likely number of unknown documents in a test for which matches existed was three.

Table 2 shows the percentage $p$ of unknown documents in our tests that had $m$ matching documents in the database packages (where $m$ in our tests ranged from 0 to 4 ). The third row of this table reads: " $13.3 \%$ of the documents in the unknown packages had exactly two matches in the corresponding database packages." 


\begin{tabular}{c|c}
\hline $\boldsymbol{m}$ matches & $\boldsymbol{p}$, percentage \\
\hline 0 & $43.3 \%$ \\
1 & $40.3 \%$ \\
2 & $13.3 \%$ \\
3 & $2.8 \%$ \\
4 & $0.3 \%$ \\
5 & $0.0 \%$ \\
\hline
\end{tabular}

Table 2: The percentage $p$ of unknown documents in our tests that had $m$ matching documents in the database packages

Finally, Table 3 shows the percentage $p$ of tests that had $m$ matching pairs of documents in the unknown and database packages. The third row of this table reads: "in $6.9 \%$ of the tests, the test-taker should have found exactly two matching pairs."

\begin{tabular}{c|c}
\hline $\boldsymbol{m}$ matching pairs & $\boldsymbol{p}$, percentage \\
\hline 0 & $0.0 \%$ \\
1 & $2.8 \%$ \\
2 & $6.9 \%$ \\
3 & $18.0 \%$ \\
4 & $21.5 \%$ \\
5 & $22.9 \%$ \\
6 & $16.7 \%$ \\
7 & $4.9 \%$ \\
8 & $3.5 \%$ \\
9 & $1.4 \%$ \\
10 & $1.4 \%$ \\
\hline
\end{tabular}

Table 3: The percentage $p$ of tests that had $m$ matching pairs

\section{The Task}


All the tests were administered personally by the first author. He provided written instructions for each test-taker and repeated them orally before the test was taken.

Test-takers received a description of the two document packages, including the statement: "these documents were obtained from willing participants who wrote naturally in a well-lit environment. To the best of our knowledge, no forgeries or disguised writings are included."

The task was described as follows: "Decide for each of the unknown documents whether or not it has a match in the database document package, and list the matches by their code numbers. By "match" we mean that you can state that the two documents were generated by the same writer using the term identification or strong probability. (We use the ninepoint ASTM standard E1658 scale of identification / strong probability / probable / indications / no conclusion / indications did not / probably did not / strong probability did not / elimination.) We ask you to tell us whether you made an identification or strong probability conclusion about each match."

The test was designed for a period of three hours but no test-taker required that long. Table 4 shows the format of the answer sheet.

A copy of the ASTM standard was available in the room where the exam was given. Instructions for professional and nonprofessional test-takers were the same, except that the first author has explicitly explained orally to each nonprofessional participant the ASTM scale and the terms "identification" and "strong probability." 


\section{Code \# of Unknown \\ Document:}

1: 111-AAA

2: $\quad$ 789-A29
Code Number of Documents that match the sample document, please indicate whether strong probability (S.P.) or identification (I.D.):
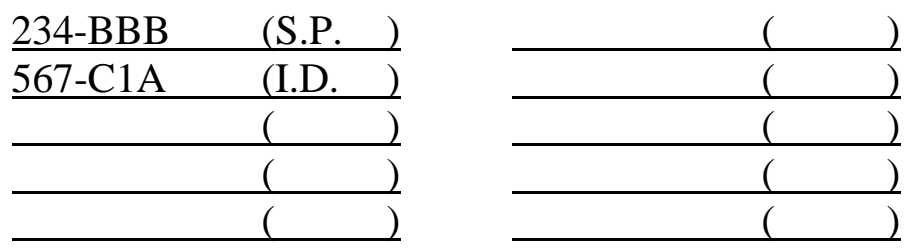

$\underline{\text { no matches }}$
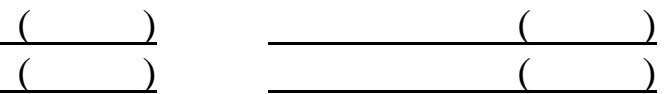

Table 4: Answer sheet format for the test

The format of the test was selected to resemble a multi-suspect case where extensive examination of documents was required. The test was designed to require a large number of comparisons on relatively short texts. In about one third of the cases, test-takers had to study document pairs that carried the same text, and in the remaining two-thirds of the cases, document pairs that carried different texts.

\section{Performance Results}

In this section we present statistics on the results from the tests. In the subsequent sections, we compare the data collected from professionals, nonprofessionals, and trainees to determine whether or not they came from genuinely different populations. We present three performance indices. The first performance index is a pair of detection/false alarm probabilities of the \{Type I; Type II $\}$ structure used in hypothesis testing ([14] [19]). The second is the average number of wrong matching declarations. The third is a Bayesian cost, representing financial reward for performance. 
Performance Index 1: Hit Rate and Wrong Association Rate

This criterion is a pair of probabilities:

- probability that a match was declared given that a match existed, (this is $\mathrm{P}\left(\mathrm{D}_{1} \mid \mathrm{H}_{1}\right)$ the probability of match detection) and,

- probability that an unknown document was wrongly matched to a database document (wrong association rate, w.a.r.; the probability of document-association false alarm).

These two probabilities are closely linked and are presented in Table 5 together. ${ }^{1}$

We show $\mathrm{P}\left(\mathrm{D}_{1} \mid \mathrm{H}_{1}\right)$, the probability of match detection in two ways:

(i) Group hit rate $=\frac{m}{n}$,

where, $m=$ number of correct matches declared by the tested group, and $n=$ number of matches that existed in the group's tests;

(ii) Average hit rate $=\frac{1}{N} \sum_{i=1}^{N} \frac{m_{i}}{n_{i}}$,

where $N=$ total number of test-takers,

$m_{i}=$ number of correct matches declared by test-taker $i$, and

$n_{i}=$ number of matches that existed in test-taker $i$ 's test.

We calculate the w.a.r. as follows:

\footnotetext{
${ }^{1}$ The higher the probability of detection (which is desirable), the higher the probability of false alarm (which is undesirable). It is easy to achieve perfect performance in either one of these probabilities - but not in both (by declaring that all unknown documents match all the database documents one gets $\mathrm{P}\left(\mathrm{D}_{1} \mid \mathrm{H}_{1}\right)=1$, but then w.a.r. $=1$; by declaring no matches at all, w.a.r. $=0$, but then $\mathrm{P}\left(\mathrm{D}_{1} \mid \mathrm{H}_{1}\right)=0$ as well.
} 
w.a.r. $=\frac{1}{N} \sum_{i=1}^{N} \frac{m_{i}}{n_{i}}$

where $N=$ total number of test-takers,

$m_{i}=$ number of unknown documents wrongly associated by test-taker $i$, and

$n_{i}=$ number of unknown documents examined by test-taker $i$.

The group hit rate and the average hit rate should be very close in groups that are homogeneous with respect to detecting a match. The ideal value for both is 1.00 . The wrong association ratio should be 0.00 ideally. In the context of our test, reluctance to match (manifested by hit rates less than 1.00) is less serious than engagement in wrong matchings (manifested by w.a.r.s greater than zero). Failure to detect a match (type II error) can be described as overcautious. Declaration of false matches (type-I error) is much more dangerous because it may link a person to an incriminating document that s/he did not create. Table 5 shows the group hit rate, average group rate, and wrong association rate for the tested groups.

\begin{tabular}{r|r|r|r} 
& \multicolumn{2}{|c|}{ Hit Rate } & \\
Group & Group & Average & w.a.r. \\
\hline Professionals-Northeast & 0.883 & 0.891 & 0.064 \\
Professionals-Southeast & 0.876 & 0.873 & 0.059 \\
Professionals-Southwest & 0.878 & 0.852 & 0.072 \\
\hline All Professionals & 0.879 & 0.871 & 0.065 \\
\hline Non-professionals & 0.877 & 0.875 & $\mathbf{0 . 3 8 3}$ \\
\hline Trainees & 0.878 & 0.852 & 0.083 \\
\hline
\end{tabular}

Table 5: Hit rate and wrong association rate

These are very interesting results. All groups have roughly the same rates of finding a match when one exists. However, the nonprofessionals are grossly over matching. 
They erroneously match unknown documents with database documents at very high rates.

In fact we have created here a Neyman-Pearson test with constant detection rate [14], and found that nonprofessionals are 6 times more likely than professionals to match two documents that were created by different writers.

\section{Performance Index 2: Absolute Number of Wrong Associations}

The first performance index counted how many unknown documents were mismatched (i.e., were paired at least once with a document not created by the same author). Thus, if one unknown document was mismatched twice, we said there that one unknown document was mismatched. In this section we count wrong matching declarations. If one unknown document was mismatched twice, we count here two wrong matching declarations. We show the average number of wrong matching declarations made by testtakers in Table 6. 


\begin{tabular}{r|c}
\hline Group & $\begin{array}{r}\text { Average number of } \\
\text { wrong associations }\end{array}$ \\
\hline Professionals-Northeast & 0.59 \\
Professionals-Southeast & 0.35 \\
Professionals-Southwest & 0.57 \\
\hline All Professionals & 0.50 \\
\hline Non-professionals & $\mathbf{5 . 8 5}$ \\
\hline Trainees & 0.50 \\
\hline
\end{tabular}

Table 6: The average number of wrong matching declarations per test-taker

The nonprofessionals are very distinct, making as many as 10 times more wrong matching declarations than the professionals.

\section{Performance Index 3: Financial Reward for Performance (Bayesian Cost)}

In the actual test nonprofessionals were paid $\$ 25$ for participation, and $\$ 25$ for each correct match. The sum of $\$ 25$ was subtracted for each incorrect match, and \$10 was subtracted for each match that was missed. If the final payment was less than $\$ 25$, the participant received $\$ 25$.

Table 7 shows what would have been paid to the participants if the guaranteed $\$ 25$ minimum payment were eliminated. This index is a particular realization of a Bayesian cost [14] with $C_{10}=-25, C_{01}=-10, C_{11}=+25$, and $C_{00}=0$. The first column shows the average payment for actual performance. The second column shows the average payment for perfect performance. The third column shows the ratio of the two. 


\begin{tabular}{r|r|r|r} 
& \multicolumn{2}{|c|}{ Payment } & Earning \\
Group & Actual & Perfect & Ratio \\
\hline Professionals-Northeast & 74.41 & 106.62 & 0.70 \\
Professionals-Southeast & 79.26 & 106.62 & 0.74 \\
Professionals-Southwest & 87.30 & 122.30 & 0.71 \\
\hline All Professionals & 80.52 & 112.14 & 0.72 \\
\hline Non-professionals & $\mathbf{- 6 1 . 8 8}$ & 101.88 & $\mathbf{- 0 . 6 1}$ \\
\hline Trainees & 93.75 & 128.13 & 0.73 \\
\hline
\end{tabular}

Table 7: Performance-based payment (Bayesian cost)

As the table demonstrates, the professionals have "earning ratios" exceeding 0.7 . The nonprofessionals, under this scheme, would have to pay a penalty.

\section{Time Spent on the Test}

Table 8 shows the average time period spent on the test and the standard deviation of this period. Nonprofessionals spent the least amount of time, followed by trainees and professionals. All participants in our test had strong incentive to test at peak performance, and all were told that they can take up to three hours to complete the test. No one required that long. We conclude that the test-takers saw no reason to use more time than they have actually used under the conditions of the test. We applied standard tests to seek correlations within groups between time spent on the test and performance, but no significant correlations were found. 


\begin{tabular}{r|r|r} 
& \multicolumn{2}{|c}{ Time Spent } \\
Group & Average & Std. Dev. \\
\hline Professionals-Northeast & $1: 23$ & $0: 32$ \\
Professionals-Southeast & $1: 34$ & $0: 36$ \\
Professionals-Southwest & $1: 38$ & $0: 20$ \\
\hline All Professionals & $1: 32$ & $0: 30$ \\
\hline Non-professionals & $0: 58$ & $0: 24$ \\
\hline Trainees & $1: 16$ & $0: 43$ \\
\hline
\end{tabular}

Table 8: Average time spent on the test and the standard deviation (hours:minutes)

\section{Comparison Criteria}

The dictionary's definition of an expert is "a person who has special skill or knowledge in some particular field" [15]. Skill or knowledge in experts is "special" only if it does not exist in the general population. In this context, it is important to compare samples created by the professionals and nonprofessionals, and determine whether or not they came from different populations with respect to handwriter-identification abilities.

\section{Statistical Tests}

The literature ([16] - [21]) offers a number of statistical tests for comparing samples, each relying on its own set of assumptions regarding sample size and statistical distributions of the data. Our study requires tests that compare data from two groups (e.g., experts vs. non-experts) and data from $k(k \geq 3)$ groups (e.g., data from the three groups of experts). In each case we use a test on distributions (of the Kolmogorov-Smirnov type), and a test on locations (of the Mann-Whitney type). Examination of the data that we compare tends 
to favor the distribution tests. We include location tests for completeness, and since some relevant past studies have used them.

Four statistical tests were used; the first two are distribution tests, the other two are location tests.

\section{Distribution Tests of the Kolmogorov-Smirnov Type}

The Kolmogorov-Smirnov (KS) two-sample test ([16, p. 127][17, section 3.9.3]) was used to decide whether or not two independent samples have been drawn from the same population (or from populations with the same distribution). This two-tailed test is "sensitive to any kind of difference in the distributions from which the two samples were drawn" [16, p. 127]. It uses the statistic

$$
D=\max _{X}\left|S_{1}(X)-S_{2}(X)\right|
$$

where

$S_{1}(X)=$ cumulative step function for the first sample which has $n_{1}$ points. It takes a value of $K / n_{1}$, where $K$ is the number of scores equal to or less than $\mathrm{X}$, and

$S_{2}(X)=$ cumulative step function for the second sample which has $n_{2}$ points. It takes a value of $K / n_{2}$, where $K$ is the number of scores equal to or less than $X$.

For values of $n_{1}$ and $n_{2}$ such that $n_{1}+n_{2}>35$, and a significance level of 0.05 , the critical value of $D$ is given by $D_{C}=1.36 \sqrt{\frac{n_{1}+n_{2}}{n_{1} n_{2}}}[17, \mathrm{p} .291]$. If $D$ is greater than $D_{C}$ then we reject the hypothesis $\mathrm{H}_{0}$ that the two samples were drawn from the same population. Otherwise, we accept this hypothesis. Alternatively, we may calculate the 
probability $p$ of obtaining, under hypothesis $\mathrm{H}_{0}$, a more extreme value of $D$ than the observed value of $D[18$, p. 624].

The Birnbaum-Hall (BH) $k$-sample test ([20, p. 382],[21]) was used to decide whether $k$ independent samples have been drawn from populations with the same distribution. It uses the statistic

$$
T=\max _{i, j, X}\left|S_{i}(X)-S_{j}(X)\right|
$$

where

$$
\begin{aligned}
& S_{i}(X)=\text { cumulative step function for the sample } i \text { (calculated as described in the } \\
& \text { Kolmogorov-Smirnov test), and } \\
& S_{j}(X)=\text { cumulative step function for the sample } j .
\end{aligned}
$$

This statistic is the maximum distance between any pair of the $k$ distribution functions. The probability $p$ of obtaining, under hypothesis $\mathrm{H}_{0}$, a larger value for the statistic than the observed value is calculated by the iterative scheme described in [21].

\section{Location Tests Based on Ranks}

The rank test of Mann and Whitney (MW) [17, section 3.9.4] was used to test whether the populations of two independent samples differ with respect to their means. The test uses the statistic

$$
\begin{gathered}
U=\min \left(U_{1}, U_{2}\right), \\
U_{1}=m n+\frac{m(m+1)}{2}-R_{1}, \\
U_{2}=m n+\frac{n(n+1)}{2}-R_{2},
\end{gathered}
$$

where, 
$m, n \quad=$ number of elements in samples 1 and 2 respectively,

$R_{l} \quad=$ sum of the ranks in group 1 , and

$R_{2} \quad=$ sum of the ranks in group 2.

The following equation is used to adjust for ties.

$$
\hat{U}=\frac{\left|U-\frac{m n}{2}\right|}{\sqrt{\left[\frac{m n}{N(N-1)}\right] \cdot\left[\frac{N^{3}-N}{12}-\sum_{i=1}^{T} \frac{\left(t_{i}^{3}-t_{i}\right)}{12}\right]}}
$$

$N \quad=$ total number of elements, $N=m+n$,

$T=$ the total number of ties observed, and

$t_{i} \quad=$ number of equal ranks in the $i$ th tie.

For large sample sizes, $\hat{U}$ is approximately normally distributed. For a significance level of 0.05 , we accept $\mathrm{H}_{0}$ if $\hat{U}<1.96$, otherwise we reject $\mathrm{H}_{0}$. We may calculate the probability $p$ of obtaining, under hypothesis $\mathrm{H}_{0}$, a more extreme value of $\hat{U}$ by using tables of the normal distribution.

The Kruskal-Wallis (KW) one-way analysis of variance by ranks ([16, chapter 8], [17, section 3.9.5]) was used to decide whether $k \geq 3$ independent samples are from different populations with respect to means. "The Kruskal-Wallis technique tests the null hypothesis that the $k$ samples come from the same population or from identical populations with respect to averages" [16, p. 184]. The Kruskal-Wallis statistic is 


$$
H=\frac{\frac{12}{N(N+1)} \sum_{j=1}^{k} \frac{R_{j}^{2}}{n_{j}}-3(N+1)}{1-\frac{\sum_{i=1}^{T}\left(t_{i}^{3}-t_{i}\right)}{N^{3}-N}},
$$

where

$k=$ number of the groups being compared,

$n_{j}=$ number of members in the $j$ th group,

$N=$ the sum of the members in all groups, i.e., $\sum_{j=1}^{k} n_{j}$,

$R_{j}=$ sum of ranks in the jth group,

$T=$ the total number of ties observed, and

$t_{i}=$ number of equal ranks in the $i$ th tie.

When the sample sizes are sufficiently large $\left(n_{j}>5\right), H$ is distributed as chi square with $d f=k-1$ degrees of freedom. At a significance level of 0.05 , the critical value $H_{C}$ for $k$ $=3$ is 5.99 and for $k=4, H_{C}=7.81$. If $H>H_{C}$, we reject the hypothesis that the samples are from the same population. Alternatively, we may calculate the probability $p$, under hypothesis $\mathrm{H}_{0}$, of obtaining a larger value for the statistic than the observed value of $H$ by using chi-square distribution tables.

\section{Scoring Criteria}

We have used five scoring criteria to compare the data obtained from the tested populations. These criteria contain and expand upon the performance indices described earlier.

Criterion 1: Number of Wrongly Associated Documents 
We have assigned to each test-taker a score based on the number of unknown documents that $\mathrm{s} /$ he has associated wrongly with database documents. The score 0 was given to examiners that associated no unknown document wrongly with a database document. The score 6 was given to examiners that wrongly associated all six unknown documents with database documents. This scoring method is in essence the w.a.r.

\section{Criterion 2: Probability that an Association Declaration is Correct}

We have scored all test-takers in terms of the ratio of correct-matching decisions to the total number of matching decisions $\left(\mathrm{P}\left(\mathrm{D}_{1} \mid \mathrm{H}_{1}\right)\right)$.

\section{Criterion 3: Hit Rate}

We scored individuals by hit rate $=\frac{m}{n}$, where $m=$ number of matches declared by the individual, and $n=$ number of matches that existed in the individual's test.

\section{Criterion 4: Earning Ratio (Based on Payment as the Bayesian Cost)}

This criterion uses the Bayesian cost. A correct match was rewarded by $\$ 25$, an incorrect match cost $\$ 25$, and a missed match cost $\$ 10$. We have scored test-takers by the ratio of the payment they have received to the payment that they would have received had they been perfectly correct ("earning ratio").

\section{Criterion 5: P-rank}

We developed a grading scheme, called a performance rank (or P-Rank) based on the different types of errors observed in our test. This scheme divides the test-takers into ten 
different sub-groups, based on the severity and number of errors observed. The grading scheme is described in Table 9.

\begin{tabular}{rr|c}
\hline Incorrect Matches & Missed Detections & Grade \\
\hline 0 & 0 & 1 \\
0 & 1 & 2 \\
0 & 2 & 3 \\
0 & $>2$ & 4 \\
1 & 0 & 5 \\
1 & 1 or more & 6 \\
2 & 0 & 7 \\
2 & 1 or more & 8 \\
3 & 0 & 9 \\
All other combinations of errors & 10 \\
\hline
\end{tabular}

Table 9: The P-rank method for assigning grades for performance

\section{Hypothesis Tested}

Using these five criteria, three hypothesis-tests were conducted. We have tested data from

(i) the three professional groups,

(ii) four groups - the group of nonprofessionals and the three groups of professionals, and

(iii) the group of all professionals and the group of nonprofessionals.

Hypothesis-test 1 (three professional groups): we tested the hypothesis that

- there is no difference in the scores collected from the three professional groups $\left(H_{0}\right)$

against the hypothesis that 
- there is a difference in the scores collected from the three professional groups $\left(H_{1}\right)$.

Hypothesis-test 2 (four groups, three professional, one nonprofessional): we tested the hypothesis that

- there is no difference in the scores collected from the three professional groups and the group of nonprofessionals $\left(H_{0}\right)$,

against the hypothesis that

- there is a difference in the scores collected from the three professional groups and the group of nonprofessionals $\left(H_{1}\right)$.

Hypothesis-test 3 (two groups, all professionals and the nonprofessionals): we tested the hypothesis that

- there is no difference in the scores collected from the group of all professionals and the group of nonprofessionals $\left(H_{0}\right)$,

against the hypothesis that

- there is a difference in the scores collected from the group of all professionals and the group of nonprofessionals $\left(H_{1}\right)$.

\section{Results for Professionals}

The results of the three hypothesis tests against the five scoring criteria using the Kolmogorov-Smirnov and location tests are given in tables 10 through 15. 


\begin{tabular}{r|rrr}
\hline $\begin{array}{r}\mathbf{H}_{\mathbf{0}}: \text { these samples are from the same } \\
\text { population using --------- }\end{array}$ & statistic & $\mathbf{p}$ & decision \\
\hline $\mathbf{7 . 2 . 1}$ - w.a.r. & 0.0541 & $\sim 1.0000^{\dagger}$ & ACCEPT \\
$\mathbf{7 . 2 . 2}$ - hit rate & 0.1471 & 0.9543 & ACCEPT \\
$\mathbf{7 . 2 . 3}-\mathbf{P}\left(\mathbf{H}_{\mathbf{1}} \mid \mathbf{D}_{\mathbf{1}}\right)$ & 0.1176 & 0.9990 & ACCEPT \\
$\mathbf{7 . 2 . 4}-$ payment & 0.1113 & 0.9993 & ACCEPT \\
$\mathbf{7 . 2 . 5}-\mathbf{P}$-rank & 0.0644 & $\sim 1.0000^{\ddagger}$ & ACCEPT \\
\hline
\end{tabular}

Table 10: Hypothesis-test 1 using the Birnbaum-Hall distribution test: should we accept the hypothesis that the samples collected from the three professional groups come from the same population?

\begin{tabular}{r|rrr}
\hline $\begin{array}{r}\mathbf{H}_{\mathbf{0}} \text { : these samples are from the same } \\
\text { population using --------- }\end{array}$ & statistic & $\mathbf{p}$ & decision \\
\hline w.a.r. & 0.0659 & 0.9676 & ACCEPT \\
hit rate & 0.2197 & 0.8960 & ACCEPT \\
$\mathbf{P}\left(\mathbf{H}_{\mathbf{1}} \mid \mathbf{D}_{\mathbf{1}}\right)$ & 0.1254 & 0.9392 & ACCEPT \\
payment & 0.3998 & 0.8188 & ACCEPT \\
P-rank & 0.7053 & 0.7028 & ACCEPT \\
\hline
\end{tabular}

Table 11: Hypothesis-test 1 using the Kruskal-Wallis location test: should we accept the hypothesis that the samples collected from the three professional groups come from the same population?

\begin{tabular}{r|rrr}
\hline $\begin{array}{r}\mathbf{H}_{\mathbf{0}} \text { : these samples are from the same } \\
\text { population using ------.r }\end{array}$ & statistic & $\mathbf{p}$ & decision \\
\hline w.a.r. & 0.5165 & $3.72 \mathrm{E}-04$ & REJECT \\
hit rate & 0.5022 & $5.47 \mathrm{E}-04$ & REJECT \\
$\mathbf{P}\left(\mathbf{H}_{\mathbf{1}} \mid \mathbf{D}_{\mathbf{1}}\right)$ & 0.4964 & $9.29 \mathrm{E}-04$ & REJECT \\
payment & 0.4534 & $3.71 \mathrm{E}-03$ & REJECT \\
P-rank & 0.5072 & $4.71 \mathrm{E}-04$ & REJECT \\
\hline
\end{tabular}

Table 12: Hypothesis-test 2 using the Birnbaum-Hall distribution test: should we accept the hypothesis that the samples collected from the three professional groups and the nonprofessional group come from the same population?

\footnotetext{
The actual value of $p$ is $1-7.25 \mathrm{E}-17$.
}

$¥$ The actual value of $p$ is $1-6.11 \mathrm{E}-10$. 


\begin{tabular}{r|rrr}
\hline $\begin{array}{r}\mathbf{H}_{\mathbf{0}} \text { : these samples are from the same } \\
\text { population using -------. }\end{array}$ & statistic & $\mathbf{p}$ & decision \\
\hline w.a.r. & 37.3169 & $3.94 \mathrm{E}-08$ & REJECT \\
hit rate & 0.3248 & $9.55 \mathrm{E}-01$ & ACCEPT \\
$\mathbf{P}\left(\mathbf{H}_{\mathbf{1}} \mid \mathbf{D}_{\mathbf{1}}\right)$ & 32.4363 & $4.23 \mathrm{E}-07$ & REJECT \\
payment & 20.9509 & $1.08 \mathrm{E}-04$ & REJECT \\
P-rank & 22.9556 & $4.13 \mathrm{E}-05$ & REJECT \\
\hline
\end{tabular}

Table 13: Hypothesis-test 2 using the Kruskal-Wallis location test: should we accept the hypothesis that the samples collected from the three professional groups and the nonprofessional group come from the same population?

\begin{tabular}{r|rrr}
\hline $\begin{array}{r}\mathbf{H}_{\mathbf{0}} \text { : these samples are from the same } \\
\text { population using --- }\end{array}$ & statistic & $\mathbf{p}$ & decision \\
\hline w.a.r. & 0.6098 & $7.23 \mathrm{E}-03$ & REJECT \\
hit rate & 0.5366 & $2.57 \mathrm{E}-02$ & REJECT \\
$\mathbf{P}\left(\mathbf{H}_{\mathbf{1}} \mid \mathbf{D}_{\mathbf{1}}\right)$ & 0.5610 & $1.71 \mathrm{E}-02$ & REJECT \\
payment & 0.5122 & $3.78 \mathrm{E}-02$ & REJECT \\
$\mathbf{P - r a n k}$ & 0.5610 & $1.71 \mathrm{E}-02$ & REJECT \\
\hline
\end{tabular}

Table 14: Hypothesis-test 3 using the Kolmogorov-Smirnov distribution test: should we accept the hypothesis that the samples collected from the professionals come from the same population as the nonprofessionals?

\begin{tabular}{r|rrr}
\hline $\begin{array}{r}\mathbf{H}_{\mathbf{0}} \text { : these samples are from the same } \\
\text { population using -- }\end{array}$ & statistic & $\mathbf{p}$ & decision \\
\hline w.a.r. & 6.1057 & $5.14 \mathrm{E}-10$ & REJECT \\
hit rate & 0.2893 & $3.86 \mathrm{E}-01$ & ACCEPT \\
$\mathbf{P}\left(\mathbf{H}_{\mathbf{1}} \mid \mathbf{D}_{\mathbf{1}}\right)$ & 5.6862 & $6.51 \mathrm{E}-09$ & REJECT \\
payment & 4.5412 & $2.80 \mathrm{E}-06$ & REJECT \\
P-rank & 4.7352 & $1.10 \mathrm{E}-06$ & REJECT \\
\hline
\end{tabular}

Table 15: Hypothesis-test 3 using the Mann-Whitney location test: should we accept the hypothesis that the samples collected from the professionals come from the same population as the nonprofessionals?

The distribution tests provide the following conclusions with respect to all five criteria:

(i) The data generated by the three professional groups came from populations that are statistically similar; 
(ii) the data generated by the three professional groups and the nonprofessional group came from populations that are statistically different;

(iii) the data generated by the professional group and nonprofessional group came from populations that are statistically different.

The same conclusions are borne out by the location tests, with the exception of the hit rate criterion. This is hardly surprising, as we already know that the average hit rate is approximately the same in all tested populations. The Mann-Whitney and Kruskal-Wallis tests, which examine ranks by averages, therefore accept the hypothesis that hit rate data of the professionals and nonprofessionals have the same averages. The KolmogorovSmirnov type tests, however, are sensitive to differences in distributions, and recognize that while the averages are the same (tables 13 and 15), the distributions are different (tables 12 and 14). Indeed the distribution tests distinguish between the hit-rate data generated by the professionals and the hit-rate data generated by nonprofessionals even though "on average" they are the same.

Notes

1. When test-takers that did not fill out the voluntary questionnaire were dropped from the professional pool there was no meaningful change in the group performance measures.

2. When nonprofessional test-takers with no college degree were dropped from the nonprofessional pool there was no meaningful change in the group performance measures. 


\section{Results for Trainees}

We compared the trainees against the group of all professional document examiners, using the five scoring methods discussed previously. Results are shown in Table 16. We also compared the trainees against the group of all nonprofessionals. These results are shown in Table 17. Since these values were obtained with a much smaller number of individuals (8 trainees), they are somewhat less statistically significant than the values reported in the previous section.

\begin{tabular}{r|ccr}
\hline $\mathbf{H}_{\mathbf{0}}$ : these samples are from the same population & statistic & $\mathbf{p}$ & decision \\
using $-\boldsymbol{w . a . r}$ & 0.488 & $7.51 \mathrm{E}-07$ & REJECT \\
hit rate & 0.362 & $5.90 \mathrm{E}-04$ & REJECT \\
$\mathbf{P}\left(\mathbf{H}_{\mathbf{1}} \mid \mathbf{D}_{\mathbf{1}}\right)$ & 0.648 & $9.94 \mathrm{E}-12$ & REJECT \\
payment & 0.486 & $6.06 \mathrm{E}-06$ & REJECT \\
P-rank & 0.469 & $2.33 \mathrm{E}-06$ & REJECT \\
\hline
\end{tabular}

Table 16: The Kolmogorov-Smirnov distribution test: should we accept the hypothesis that the samples collected from the trainees come from the same population as the professionals?

\begin{tabular}{r|ccr}
\hline $\mathbf{H}_{\mathbf{0}}$ : these samples are from the same population & statistic & $\mathbf{p}$ & decision \\
$\mathbf{u}$ using ------ & & & \\
\hline w.a.r. & 0.610 & 0.007 & REJECT \\
hit rate & 0.537 & 0.026 & REJECT \\
$\mathbf{P}\left(\mathbf{H}_{\mathbf{1}} \mid \mathbf{D}_{\mathbf{1}}\right)$ & 0.561 & 0.017 & REJECT \\
payment & 0.512 & 0.038 & REJECT \\
P-rank & 0.561 & 0.017 & REJECT \\
\hline
\end{tabular}

Table 17: The Kolmogorov-Smirnov distribution test: should we accept the hypothesis that the samples collected from the trainees come from the same population as the nonprofessionals? 
It is apparent that the score distributions of the trainees' differ from both the distributions of the professionals and the nonprofessionals. At this stage the trainees' data, as judged by values of $p$ in tables 16 and 17, are somewhat "closer" to that of the nonprofessionals than to that of the professionals. We indeed expect trainees to be "between" nonprofessionals and professionals. It would be interesting to test the same trainee group in a year or two and see if its performance "moved" toward that of the professional group.

\section{Concluding Remarks}

- Test results lay to rest the debate over whether or not professional document examiners possess writer-identification skills absent in the general population. They do.

- Professional document examiners were capable of excellent performance in our tests - especially in avoiding wrong matching of documents to each other - even without the benefit of laboratory equipment or consultation with colleagues (that are available in practice).

- Lay persons are deficient as document examiners because they tend to over-match documents by declaring that documents generated by different people have come from the same hand.

- The data collected may provide answers to several additional interesting questions relating to proficiency. Is there a common property that characterizes the few errors made by the professional document examiners? Is there correlation between length of professional experience (or other factors) and performance? Are there sub-groups of examiners whose training, professional affiliations, or court experience make them more proficient than other groups? How important were the similarity/dissimilarity of texts and the lengths of texts in affecting performance? 
- During the preparation of the test, we created a large corpus of original documents that could be used as a database for training, testing, machine intelligence studies, and further development of standards in writer identification.

\section{References}

1. Risinger DM, Denbeaux MP, Saks MJ. Exorcism of Ignorance as a Proxy for Rational Knowledge: the Lessons of Handwriting Identification 'Expertise'. University of Pennsylvania Law Review 1989; 137: 731-787.

2. Kam M, Wetstein J, and Conn R. Proficiency of Professional Document Examiners in Writer Identification. J Forensic Sci 1994; 39: 5-14.

3. Galbraith O, Galbraith CS, Galbraith NG. The Principle of the "Drunkard's Search" as a Proxy for Scientific Analysis: The Misuse of Handwriting Test Data in a Law Journal Article. Intl J Forensic Doc Exam 1995; 1(1): 7-17.

4. Beck, J. Sources of Errors in Forensic Handwriting Evaluation. J Forensic Sci 1995; 40(1): 78-82.

5. United States of America against Roberta Starzecpyzel and Eileen Starzecpyzel United States District Court, Southern District of New York, case no. S1:93 CR.49.

6. United States of America against Kathleen Kresner Jones, United States Federal District Court, Eastern District of Tennessee, case no. 3:95 CR.24.

7. United States vs. Specialist Jeffery A. Ruth, US Army Court of Criminal Appeals, case no. 9400093.

8. United States of America; Government of the Virgin Islands v. Edwin Velasquez, United States Court of Appeals, Third District, case no. 93-7236.

9. United States of America vs. Ruben Renteria Sr. and Ruben Renteria Jr., United States District Court for the District of New Mexico, case no. 95-320-JP. 
10. United States of America vs. Joseph Pravato and Syed Arrhad Mahmood, United States District Court, Eastern District of New York, case no. 95 CR 981.

11. United States of America vs. Muhammed Ijaz Chohan, United States District Court, Eastern District of New York, case no. 95 CR 876.

12. The People of the State of California vs. Victor Kiet Diep and Thuan Wu, Superior Court of the State of California, San Francisco, court no. 157436-01/02.

13. American Society for Testing and Materials, ASTM Standard E1658: Terminology for Expressing Conclusions of Forensic Document Examiners.

14. Van Trees HL. Detection, Estimation, and Modulation Theory, Part 1. New York: Wiley, 1968.

15. The Random House Dictionary of the English Language, New York: Random House, 1987.

16. Siegel S. Nonparametric Statistics for the Behavioral Sciences. New York: McGraw Hill, 1956; 127-136, 184-194.

17. Sachs L. Applied Statistics - A Handbook of Techniques. New York: Springer Verlag, 1984.

18. Press WH, Teukolsky SA, Vetterling WT, Flannery BP. Numerical Recipes in C. New York: Cambridge University Press, 1992; 623-628.

19. Gibbons JD. Nonparametric Methods for Quantitative Analysis. New York: Holt, Rinehart and Winston, 1976.

20. Conover WJ. Practical Non-Parametric Statistics. New York: John Wiley and Sons, 1980.

21. Birnbaum ZW, Hall RA. Small sample distributions for multi-sample statistics of the Smirnov Type. Ann Math Stat 1960; 31: 710-720. 
Additional Information and Reprint Requests

\author{
Moshe Kam, Ph.D. \\ Data Fusion Laboratory \\ Electrical and Computer Engineering Department, \\ Drexel University \\ $32^{\text {nd }} \&$ Chestnut Sts. \\ Philadelphia, PA 19104
}

